\title{
Destruction of interference by many-body interactions in cold atomic Bose gases
}

\author{
S. Chen and R. Egger \\ Institut für Theoretische Physik, Heinrich-Heine-Universität, D-40225 Düsseldorf, Germany
}

(Dated: November 23, 2018)

\begin{abstract}
We study the effects of many-body interactions on the interference in a Mach-Zehnder interferometer for matter waves of ultracold Bose atoms. After switching off an axial trapping potential, the thermal initial wavepacket expands, and subsequently interference fringes may be observed in a circular 1D trap. These are computed for axial harmonic or $\delta$-function traps, and for interaction strengths from the Thomas-Fermi regime to the Tonks-Girardeau limit. It is shown that manybody correlations in a realistic setup destroy interference to a large degree. Analytical expressions allowing to infer the observability of phase coherence and interference are provided.
\end{abstract}

PACS numbers: 03.75.Kk, 03.75.-b, 05.30.Jp

\section{INTRODUCTION}

Recent experimental advances in the control of ultracold atomic gases have opened new exciting possibilities to systematically study the effects of many-body interactions in low-dimensional strongly correlated mesoscopic systems [1, 2, 3]. Two main experimental routes are presently being pursued, namely magnetic or magnetooptical trap technology $[3,4,[5,6]$, and the formation of atomic waveguides on microchips $[7,8,6$, 10]. A particularly interesting limit arises in the true 1D case, which may, for instance, be realized by confining $N$ atoms in a highly anisotropic cigar-shaped trap with large transverse trapping frequency $\omega_{\perp}$ [11, 12]. In this paper we study interacting 1D Bose gases, but similar ideas and conclusions apply to cold fermionic gases as well. In the 1D case, in an infinitely long system, quantum fluctuations prevent the existence of a Bose-Einstein condensate (BEC) even at $T=0$. For a finite system and very weak interactions, however, BEC could happen at extremely low temperatures not reachable at present 13, 14, 15, 16, 17]. The physics of a trapped 1D atomic Bose gas is then characterized by a crossover from the high-density weakly correlated Thomas-Fermi (TF) regime to a strongly correlated Tonks-Girardeau (TG) regime 18, 19] at low density, see Refs. 15, 20, 21, 22]. The relevant dimensionless interaction parameter governing this crossover in a uniform system is

$$
\gamma=m g / \hbar^{2} \rho_{0},
$$

where $g$ is an effective 1D interaction constant 23], see Eq. (3) below, and $\rho_{0}$ is the density. While for the uniform system, a Bethe ansatz solution due to Lieb and Liniger [24] is available, for the case of a (possibly time-dependent) axial trapping potential $V(x, t)$, no exact statements are known, and one generally resorts to approximate or numerical methods [25, 26, 27]. We mention in passing that this system also has very interesting single-particle excitations with exotic properties such as fractional (anyon) statistics. While such systems could also be realized in more conventional condensed matter systems, the high quality and tunability of atomic Bose gases renders them very attractive for fundamental studies.

In this paper, we address the influence of interactions on the phase coherence and the interference properties of a 1D Bose gas. To be specific, we consider the circular geometry indicated in Fig. 1 At time $t=0$, the axial trapping potential $V(x, t)$ localized around $x \approx 0$ is switched off, and after some characteristic expansion time, the expanding right- and left-moving wave packets will then meet at the other side of the ring, $x \approx \pm L / 2$. If phase coherence of the wave packets is not lost during the propagation, a Mach-Zehnder-type interference signal should be observable at this side. While a modified Gross-Pitaevskii (GP) approach, obtained from combining the Lieb-Liniger solution with a local density approximation (LDA) valid for large enough systems [20], is able to yield accurate ground-state density profiles $\rho_{0}(x, t)$ of a 1D Bose gas after switching off the axial trap even in the Tonks limit [25], it typically overestimates interference signals by orders of magnitude [26]. For a correct description of interference, it is thus necessary to take into account quantum-mechanical phase fluctuations, which have so far only been addressed in the static case 28, 29] or in the Tonks limit under rather special initial conditions 30, 31]. Below we show that under realistic modelling of the initial preparation and the subsequent dynamics, phase coherence and interference are strongly reduced by many-body interactions in 1D Bose gases. Moreover, we provide quantitative expressions to assess this effect in experiments. Proper insight into this question is of practical importance for the development of future atom interferometers and for the understanding of current experiments [10, 32, 33]. The remainder of the paper is organized as follows. In Sec. [II we study magneto-optical or magnetic traps with

$$
V(x, t)=\frac{1}{2} m \omega_{x}^{2} x^{2} \Theta(-t),
$$

with angular frequencies $\omega_{x} \ll \omega_{\perp}$; here $\Theta$ is the Heaviside function. In Sec. [II the validity of LDA is presumed. In Sec. III we briefly discuss a related setup employing a $\delta$-trap which may be more realistic for microchiptrapped gases yet allows for an exact solution of the time- 


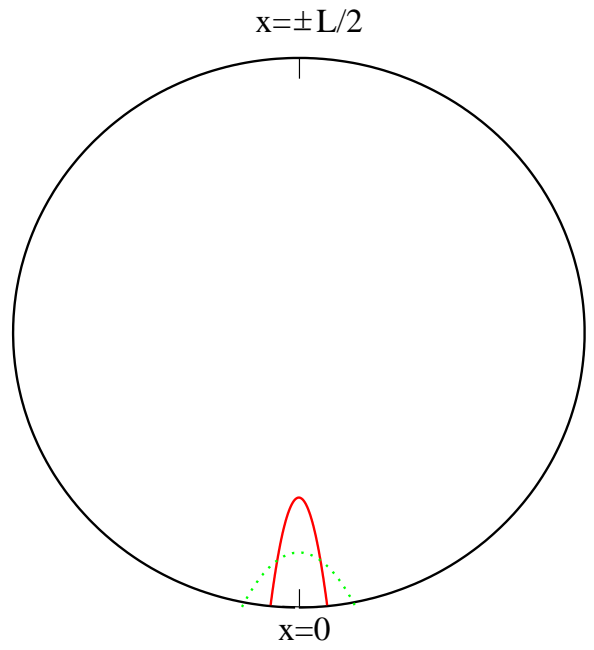

FIG. 1: Schematic geometry of a Mach-Zehnder interferometer realized in a circular trap with circumference $L$. By applying an axial trap potential at $t<0$ around $x \approx 0$, a thermal non-equilibrium initial state is prepared, whose time evolution and interference at $x \approx \pm L / 2$ are the subject of this paper.

dependent problem. Finally, we conclude in Sec. IV

\section{INTERFERENCE SIGNAL: HARMONIC INITIAL TRAP}

Let us consider a circular trap at ultralow temperature, $k_{B} T \ll \hbar \omega_{\perp}$, such that motion in the transverse direction is frozen out, see Fig. 11 The effective 1D interaction strength is 23]

$$
g=-\frac{2 \hbar^{2}}{m a_{1 \mathrm{D}}}, \quad a_{1 \mathrm{D}}=-\frac{d_{\perp}^{2}}{2 a_{s}}\left[1-\mathcal{C}\left(a_{s} / d_{\perp}\right)\right],
$$

where $a_{s}>0$ is the $3 \mathrm{D} s$-wave scattering length describing the repulsive atom-atom interactions, $d_{\perp}=$ $\left(2 \hbar / m \omega_{\perp}\right)^{1 / 2}$, and $\mathcal{C} \approx 1.4603$. The interaction strength can be tuned by Feshbach resonances [34] or by using optical lattices [2].

\section{A. Fluctuation modes}

The resulting Hamiltonian of the 1D Bose gas at fixed particle number $N$, including an arbitrary timedependent axial trap potential $V(x, t)$, is

$$
\begin{aligned}
H(t) & =\int_{-L / 2}^{L / 2} d x \hat{\psi}^{\dagger}(x, t)\left[-\frac{\hbar^{2}}{2 m} \partial_{x}^{2}+V(x, t)\right] \hat{\psi}(x, t) \\
& +\frac{g}{2} \int d x:\left[\hat{\psi}^{\dagger}(x, t) \hat{\psi}(x, t)\right]^{2}:
\end{aligned}
$$

where the colons denote normal-ordering. For $V(x, t)=$ 0 , this model was solved exactly by Lieb and Liniger [24]. To make progress in the presence of the external potential, the field operator $\hat{\psi}$ is expressed in terms of the density $\rho(x)$ and the phase $\phi(x)$,

$$
\hat{\psi}(x)=\sqrt{\rho(x)} e^{i \phi(x)} .
$$

We employ canonically conjugate density [phase] fluctuation operators $\Pi(x, t)=\rho(x, t)-\rho_{0}(x, t)[\Phi(x, t)=$ $\left.\phi(x, t)-\phi_{0}(x, t)\right]$ describing quantum fluctuations around the solution $\psi_{0}(x, t)=\sqrt{\rho_{0}(x, t)} \exp \left[i \phi_{0}(x, t)\right]$ of the time-dependent modified GP equation [20, 25],

$i \hbar \partial_{t} \psi_{0}(x, t)=\left[-\frac{\hbar^{2}}{2 m} \partial_{x}^{2}+V(x, t)+\tilde{F}\left(\rho_{0}(x, t)\right)\right] \psi_{0}(x, t)$,

where $\tilde{F}\left(\rho_{0}\right)$ is the (exactly known) chemical potential for the Lieb-Liniger problem [20], with limiting values

$$
\tilde{F}\left(\rho_{0}\right)=\left\{\begin{array}{cc}
g \rho_{0}, & \gamma \ll 1 \\
\pi^{2} \hbar^{2} \rho_{0}^{2} / 2 m, & \gamma \gg 1 .
\end{array}\right.
$$

For arbitrary time-dependent potential $V(x, t)$, no closed solution of Eq. (6) for arbitrary $\gamma$ is available. From numerical work, however, the expansion of a 1D Bose gas after switching off an harmonic axial trap potential has recently been shown to violate self-similarity 25 in the intermediate regime between the TF and the TG limit, $\gamma \approx 1$, which can be traced back to the variable nonlinearity of $\tilde{F}\left(\rho_{0}\right)$ for different $\gamma$.

Substituting Eq. (5) into Eq. (4) and expanding up to quadratic order in $\Phi$ and $\Pi$, we obtain the effective low-energy Hamiltonian describing quantum fluctuations around the solution to Eq. (6). Neglecting terms containing $\Pi \partial_{x}^{2} \Pi$ or $\left(\rho_{0}^{-1} \partial_{x}^{2} \rho_{0}\right) \Pi^{2}$, we obtain the time-dependent Hamiltonian

$$
\begin{aligned}
H(t) & =\int d x\left[\frac{\hbar^{2} \rho_{0}}{2 m}\left(\partial_{x} \Phi\right)^{2}\right. \\
& \left.+\frac{\hbar^{2}}{m}\left(\partial_{x} \phi_{0}\right) \Pi \partial_{x} \Phi+\frac{1}{2} \frac{\partial \tilde{F}\left(\rho_{0}\right)}{\partial \rho_{0}} \Pi^{2}\right] .
\end{aligned}
$$

While terms involving $\Pi \partial_{x}^{2} \Pi$ are irrelevant in the longwavelength low-energy regime, the neglect of terms $\propto$ $\left(\rho_{0}^{-1} \partial_{x}^{2} \rho_{0}\right) \Pi^{2}$ requires that the interaction strength $g$ is not too weak [35]. In practice, this approximation is valid to high accuracy even in the TF regime, and therefore in all cases of interest here. Interference fringes are correctly captured in such a long-wavelength theory despite their rapidly oscillating character, since the oscillations arise due to a mixing of left- and right-moving wave-packets. These wave-packets are both correctly described within the present theory.

Equation (8) resembles the Hamiltonian of a Luttinger liquid [13], generalized to a time-dependent and spatially non-uniform system, where a non-standard coupling between $\Pi$ and $\partial_{x} \Phi$ is induced by the time dependence of the external potential. The Hamiltonian (8) is quadratic 
in the fluctuation operators and can therefore be diagonalized by a Bogoliubov-de Gennes transformation. However, explicit diagonalization is rather difficult for arbitrary potential $V(x, t)$, and here we shall limit ourselves to the form given in Eq. (2). Although the full Hamiltonian (4) is time-independent for $t>0$, the Hamiltonian (8) governing quantum fluctuations becomes explicitly time-dependent due to the time dependence of $\rho_{0}(x, t)$ and $\phi_{0}(x, t)$. Furthermore, to make progress, we restrict ourselves to the self-similar limits $\gamma \ll 1$ and $\gamma \gg 1$. Then the density $\rho_{0}(x, t>0)$ is self-similar with some scale factor $b(t)$ [25],

$$
\rho_{0}(x, t)=\frac{n(x / b(t))}{b(t)}
$$

where $b(0)=1$ and $n(x)=\rho_{0}(x, 0)$ is the initial density profile. Solving Eq. (6) with Eq. (9) results in the phase

$$
\phi_{0}(x, t)=\frac{m x^{2}}{2 \hbar} \frac{\dot{b}(t)}{b(t)} .
$$

Under self-similarity, it is then possible to diagonalize the quadratic Hamiltonian (8) by solving the equation of motion generated by it. Using the long derivative

$$
\hat{D}=\partial_{t}+x(\dot{b} / b) \partial_{x}
$$

some algebra shows that the equation of motion for $\Phi$ is

$$
\left(\hat{D}+\alpha \frac{\dot{b}}{b}\right) \hat{D} \Phi(x, t)=\frac{1}{m} \frac{\partial \tilde{F}\left(\rho_{0}\right)}{\rho_{0}} \partial_{x}\left[\rho_{0} \partial_{x} \Phi\right]
$$

where $\alpha=1$ in the TF regime and $\alpha=2$ in the TG regime. To solve Eq. (12), we use the ansatz

$$
\Phi(x, t)=\sum_{j} C_{j}(t) f_{j}(x / b(t)) \hat{B}_{j}(t)+\text { H.c. },
$$

where $\hat{B}_{j}^{\dagger}, \hat{B}_{j}$ are standard Bose operators with time dependence governed by time-dependent eigenfrequency $\Omega_{j}(t)$,

$$
\hat{B}_{j}(t)=\exp \left[-i \int_{0}^{t} d t^{\prime} \Omega_{j}\left(t^{\prime}\right)\right] \hat{B}_{j}(0) .
$$

Furthermore, $f_{j}$ are suitably normalized eigenfunctions, and the prefactor $C_{j}$ is needed to bring $H(t)$ into the canonical form

$$
H(t)=\sum_{j} \hbar \Omega_{j}(t) \hat{B}_{j}^{\dagger}(t) \hat{B}_{j}(t),
$$

where the summation extends over all eigenmodes. One finds a similar expression to Eq. (13) for the conjugate field $\Pi$ by using the commutation relation with $\Phi$. Given the scale-factor $b(t)$ and the initial density $n(x)$ entering Eq. (9), solving the eigenproblem (12) thus leads to a complete description of the time-dependent quantum phase fluctuations. This program is carried out in this section separately for the TF limit, see Sec.II and the TG regime, see Sec. IC

To investigate coherence properties during the selfsimilar expansion process, we calculate the (equal-time) density matrix

$$
W\left(x, x^{\prime}, t\right)=\left\langle\hat{\psi}^{\dagger}(x, t) \hat{\psi}\left(x^{\prime}, t\right)\right\rangle .
$$

Following Refs. [15, 36], here it is justified to neglect density fluctations against phase fluctuations, resulting in

$$
\begin{aligned}
W\left(x, x^{\prime}, t\right) & \simeq W_{0}\left(x, x^{\prime}, t\right) e^{-F\left(x, x^{\prime}, t\right)} \\
W_{0}\left(x, x^{\prime}, t\right) & =\sqrt{\rho_{0}(x, t) \rho_{0}\left(x^{\prime}, t\right)} e^{-i\left[\phi_{0}(x, t)-\phi_{0}\left(x^{\prime}, t\right)\right]}, \\
F\left(x, x^{\prime}, t\right) & =\left\langle\left[\Phi(x, t)-\Phi\left(x^{\prime}, t\right)\right]^{2}\right\rangle / 2
\end{aligned}
$$

The task is then to compute the fluctuation correlator $F$. Substituting Eq. (13) into $F\left(x, x^{\prime}, t\right)$, it follows that

$$
\begin{aligned}
F\left(x, x^{\prime}, t\right) & =\sum_{j=0}^{\infty} \frac{1}{2}\left|C_{j}(t)\right|^{2}\left(1+2 n_{j}\right)\left[f_{j}(x, t)-f_{j}\left(x^{\prime}, t\right)\right]^{2}, \\
& =\sum_{j=0}^{\infty}\left|C_{j}(t)\right|^{2} \operatorname{coth}\left[\hbar \Omega_{j}(t) / 2 k_{B} T\right] \\
& \times \frac{1}{2}\left[f_{j}(x, t)-f_{j}\left(x^{\prime}, t\right)\right]^{2},
\end{aligned}
$$

with the Bose-Einstein distribution $n_{j}=\left\langle\hat{B}_{j}^{\dagger} \hat{B}_{j}\right\rangle=$ $\left[\exp \left(\hbar \Omega_{j}(t) / k_{B} T\right)-1\right]^{-1}$.

To analyze interference fringes, it is convenient to switch to $y=x-L / 2(y=x+L / 2)$ for $L / 2>x>0$ $(-L / 2<x<0)$, such that the center of the overlapping clouds is at $y=0$, and the detection signal is given by

$$
\begin{aligned}
I(y, t) & =\langle\rho(y+L / 2, t)\rangle+\langle\rho(-y+L / 2, t)\rangle \\
& +2 \operatorname{Re} W(y+L / 2,-y+L / 2, t) .
\end{aligned}
$$

The last term corresponds to the (first-order) interference signal $I_{\text {int }}(y)$ of interest here. Using Eq. (17), it reads

$$
\begin{aligned}
I_{\mathrm{int}}(y) & =2 \cos [2 Q y] e^{-F(y+L / 2,-y+L / 2, t)} \\
& \times \sqrt{\rho_{0}(y+L / 2, t) \rho_{0}(-y+L / 2, t)},
\end{aligned}
$$

with time-dependent modulation wavevector $Q=\left[\phi_{0}(y+\right.$ $\left.L / 2, t)-\phi_{0}(-y+L / 2, t)\right] / 2 y$, which for $|y| \ll L$ takes the form

$$
Q(t)=\partial_{x} \phi_{0}(L / 2, t)=\frac{m L}{2 \hbar} \frac{\dot{b}}{b}
$$

It is obvious from Eq. (19) that phase fluctuations entering $F\left(x, x^{\prime}, t\right)$ suppress interference.

\section{B. Thomas-Fermi regime}

In this subsection, we assume that the initial trapped gas (at $t<0$ ) is within the $\mathrm{TF}$ regime, and also stays 
within this regime throughout all relevant timescales. Then the initial density is given by 37

$$
n(x)=\frac{m}{2 g} \omega_{x}^{2}\left(R^{2}-x^{2}\right) \Theta\left(R^{2}-x^{2}\right),
$$

where the TF radius $R$ follows from $\int n(x) d x=N$,

$$
R=\left(3 N l_{x}^{4} /\left|a_{1 \mathrm{D}}\right|\right)^{1 / 3}, \quad l_{x}=\sqrt{\hbar / m \omega_{x}} .
$$

Provided the expanding gas indeed stays in the TF regime, the expansion is well described by the self-similar profile (9), where $b(t)$ is the solution to [37, 38]

$$
\ddot{b}=\omega_{x}^{2} / b^{2}, \quad \text { with } b(0)=1, \dot{b}(0)=0 .
$$

The phase $\phi_{0}(x, t)$ is then given by Eq. (10). While the eigenproblem for the quantum fluctuations has previously been solved in the static TF case [15, 35, 36, 39, 40], the coupling of $\partial_{x} \Phi$ and $\Pi$ in the time-dependent case, see Eq. (8), complicates the analysis considerably. Remarkably, it is nevertheless possible to solve the full dynamical problem analytically.

The equation of motion (12) is solved under the ansatz (13) with

$$
C_{j}(t)=\left[2 \hbar R \Omega_{j}(t) b(t) / g\right]^{-1 / 2} .
$$

To see this, we note that the long derivative (11) acts on the part belonging to the annihilation operator $\hat{B}_{j}$ in Eq. (13) as

$$
\hat{D} \Phi_{j}=\left(-i \Omega_{j}-\frac{\dot{b}}{2 b}-\frac{\dot{\Omega_{j}}}{2 \Omega_{j}}\right) \Phi_{j}
$$

After some algebra, using $y=x / R b(t)$, Eq. (12) then takes the form

$$
\frac{G_{j}}{\omega_{x}^{2}} f_{j}(y)+\frac{d}{d y}\left[\left(1-y^{2}\right) \frac{d}{d y} f_{j}(y)\right]=0,
$$

where $G_{j}(t)$ is given by

$G_{j}=b^{3}\left(\Omega_{j}^{2}-\frac{3}{4}\left(\dot{\Omega}_{j} / \Omega_{j}\right)^{2}+\ddot{\Omega}_{j} / 2 \Omega_{j}-(\dot{b} / 2 b)^{2}+\ddot{b} / 2 b\right)$.

Equation (25) has a standard solution in terms of Legendre polynomials $P_{j}$, with normalized eigenfunctions

$$
f_{j}(y)=\sqrt{j+1 / 2} P_{j}(y), \quad \int_{-1}^{1} d y F_{j}^{2}(y)=1,
$$

iff $G_{j}(t)$ is time-independent and given by

$$
G_{j}\left[\Omega_{j}(t)\right]=j(j+1) \omega_{x}^{2},
$$

where $j$ is a non-negative integer. Eigenfrequencies $\Omega_{j}(t)$ thus follow as solutions to the differential equation induced by Eq. (28). On not too long time scales, Eq. (28) with (26) has the solution

$$
\frac{\Omega_{j}(t)}{\omega_{x}}=\sqrt{\frac{2 j(j+1)+1}{4 b^{3}(t)}},
$$

which holds as long as $\dot{b}(t) / 2 b(t) \ll \Omega_{j}(t)$. For the results shown below in Figs. 2 and 3 this condition is accurately fulfilled. On much longer time scales, one should instead (numerically) solve Eq. (28). The fluctuation correlations (17) and the interference signal (19) then follow immediately using these results. It is obvious that $C_{j}(t) \propto g^{1 / 2}$, and therefore the interaction parameter $g$ enters the fluctuation correlator $F$ and suppresses the interference in an exponential manner. The validity of the self-similar TF solution and the subsequent treatment of the quantum fluctuation modes is guaranteed by the validity of $\tilde{F}\left(\rho_{0}\right)=g \rho_{0}$, which in turn is accurate to $1 \%$ for $\gamma<0.05$ [24].

To give concrete examples, consider a system of $N=$ $10^{3}{ }^{23} \mathrm{Na}$ atoms on a ring of circumference $L=16 R$, where $R$ is the TF radius. The axial trap potential is switched off at $t=0$, and we study the resulting interference signal. A qualitative estimate for the effective interaction strength at time $t>0$ can be given in terms of $\gamma(t)=2 /\left[\left|a_{1 D}\right| \rho_{m}(t)\right][20,25]$, where $\rho_{m}(t)=\rho_{0}(x=$ $0, t)$. Let us first consider a system with trap frequencies $\omega_{x}=0.5 \mathrm{kHz}$ and $\omega_{\perp}=50 \mathrm{kHz}$, where $\gamma(0) \approx 0.0006$ indicates that the system is deeply in the $\mathrm{TF}$ regime and phase fluctuations are very small. The expanding wave packets begin to meet at time $t=13 \mathrm{~ms}$. We calculated the phase fluctuations from Eq. (18) with a UV cutoff $\hbar \Omega_{j}<\mu$ given by the $t=0 \mathrm{TF}$ chemical potential, $\mu=m \omega_{x}^{2} R^{2} / 2$, see Eq. (22). In Fig. 2 the resulting interference fringes at time $t=16 \mathrm{~ms}$ corresponding to $b(t)=10$ are shown. While there is a detectable interference signal at $T=4 \mathrm{nK}$, interference is completely washed out by thermal fluctuations at $10 \mathrm{nK}$. As second example, consider the same system but with higher trap frequencies, $\omega_{x}=1 \mathrm{kHz}$ and $\omega_{\perp}=100 \mathrm{kHz}$, see Fig. 3 . Here we have stronger interactions, $\gamma(0) \approx 0.001$, but the system still stays in the $\mathrm{TF}$ regime on the timescales of interest. Clearly, pronounced interference patterns can be observed again.

\section{Tonks-Girardeau regime}

When the system leaves the TF regime during the expansion process, self-similarity is violated [25], and the solution of Eq. (12) is much more complicated. However, analytical progress is possible in the opposite TG limit. Assuming that one starts with an initial trap corresponding to the TG regime, during the subsequent expansion one will also stay in this regime, and the density becomes self-similar again [20], see Eq. (9) with $b(t)=\sqrt{1+\omega_{x}^{2} t^{2}}$ and

$$
n(x)=n_{\text {Tonks }}^{0} \sqrt{1-x^{2} / R_{0}^{2}},
$$

where $n_{\text {Tonks }}^{0}=2 N / \pi R_{0}$ and

$$
R_{0}=\sqrt{2 N \hbar / m \omega_{x}}
$$

denotes the Tonks radius. Furthermore, Eq. (10) specifies $\phi_{0}(x, t)$ again. 


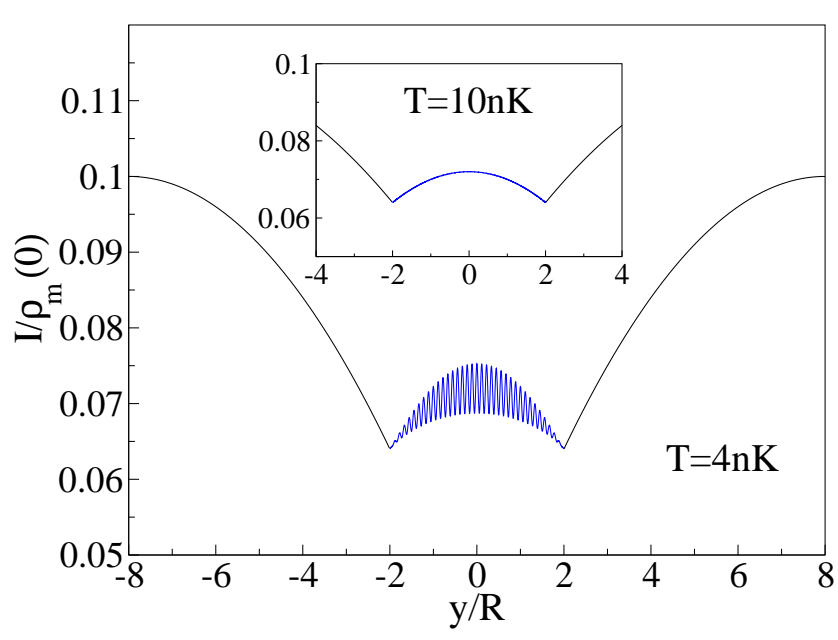

FIG. 2: The scaled density profile in units of $\rho_{m}(0)$ for $N=$ $10^{3}$ expanding $\mathrm{Na}$ atoms at time $t=16 \mathrm{~ms}$ and temperature $T=4 \mathrm{nK}$. Trap frequencies are $\omega_{x}=0.5 \mathrm{kHz}$ and $\omega_{\perp}=$ $50 \mathrm{kHz}$. The inset shows the corresponding density profile at $T=10 \mathrm{nK}$.

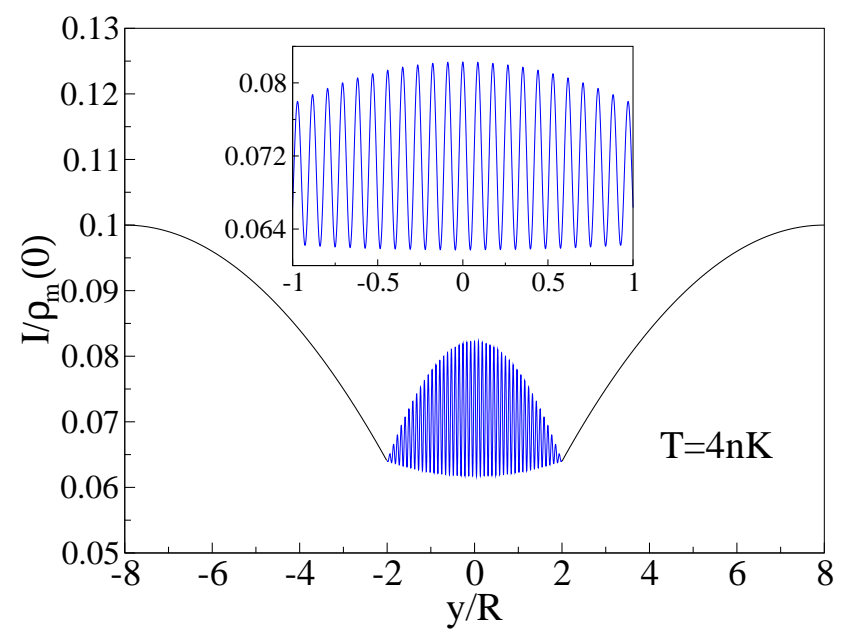

FIG. 3: Same as Fig. 2] but with $\omega_{x}=1 \mathrm{kHz}$ and $\omega_{\perp}=$ $100 \mathrm{kHz}$. The expanding clouds meet at time $t=6.5 \mathrm{~ms}$, the snapshot is shown for $t=8 \mathrm{~ms}$. The inset shows details of the interference fringes.

Now Eq. (12) can be solved by the ansatz (13) with

$$
C_{j}(t)=\left[2 \hbar m R_{0} \Omega_{j}(t) b^{2}(t) / \pi^{2} \hbar^{2} n_{\text {Tonks }}^{0}\right]^{-1 / 2} .
$$

Proceeding in the same spirit as above, using $y=$ $x / R_{0} b(t)$, Eq. (12) leads to

$$
\frac{\tilde{G}_{j}}{\omega_{x}^{2}} f_{j}(y)+\left(1-y^{2}\right) \frac{d^{2}}{d y^{2}} f_{j}(y)-y \frac{d}{d y} f_{j}(y)=0
$$

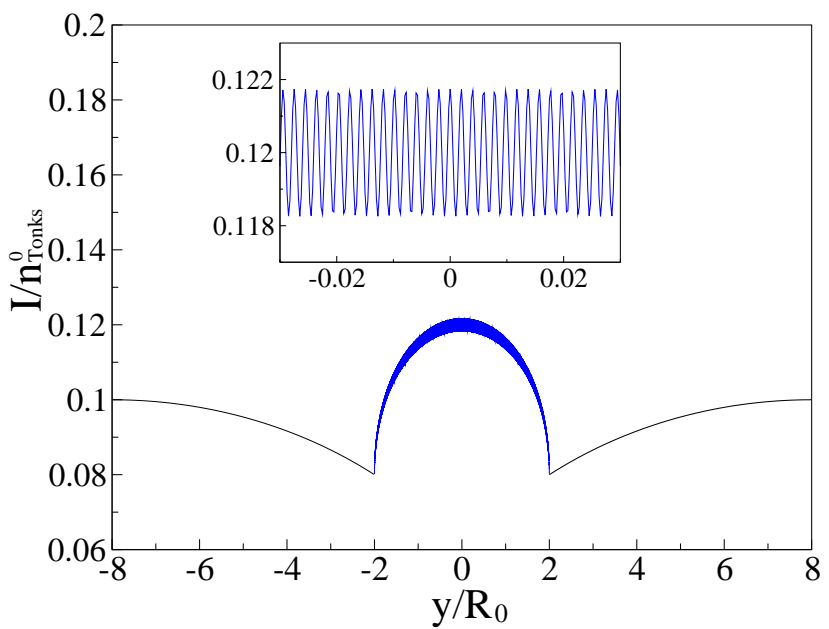

FIG. 4: The scaled density profile in units of $n_{\text {Tonks }}^{0}$ for $N=$ $10^{3}$ expanding ${ }^{87} \mathrm{Rb}$ atoms at time $t=1 \mathrm{~s}$ (see text). The inset shows details of the interference fringes.

where

$$
\tilde{G}_{j}=b^{4}\left(\Omega_{j}^{2}-\frac{3}{4}\left(\dot{\Omega}_{j} / \Omega_{j}\right)^{2}+\ddot{\Omega}_{j} / 2 \Omega_{j}+\ddot{b} / b\right) .
$$

With $j$ denoting positive integers, Eq. (33) has a solution provided that

$$
\tilde{G}_{j}=j^{2} \omega_{x}^{2}
$$

which gives a differential equation determining $\Omega_{j}(t)$. The solution to Eq. (33) is then

$$
f_{j}(y)=\sqrt{\frac{2 j \Gamma(j+1) \Gamma(j)}{\Gamma^{2}(j+1 / 2)}} P_{j}^{(-1 / 2,-1 / 2)}(y),
$$

where $P_{j}^{(-1 / 2,-1 / 2)}$ are Jacobi polynomials, and the normalization condition is

$$
\int_{-1}^{1} d y\left(1-y^{2}\right)^{-1 / 2} f_{j}^{2}(y)=1
$$

Amazingly, Eq. (35) is solved by the simple form

$$
\Omega_{j}(t) / \omega_{x}=j / b^{2}(t),
$$

which holds on all timescales. It is then straightforward to obtain the fluctuation correlations from Eq. (18), and the interference signal from Eq. (19).

Notably, even at zero temperature, interference is practically completely suppressed by many-body interactions. In Fig. [4 we show the results of the above calculation for $N=10^{3}{ }^{87} \mathrm{Rb}$ atoms on a ring of circumference $L=16 R_{0}$, where we assume a scattering length $a_{s}=42.32 \mathrm{~nm}$, e.g. resulting from tuning via a Feshbach resonance. Trap frequencies are $\omega_{x}=10 \mathrm{~Hz}$ and 
$\omega_{\perp}=100 \mathrm{kHz}$, which puts the system well into the Tonks regime, $\gamma(0) \approx 14.3$. At $t=0.8 \mathrm{~s}$ the expanding wavepackets begin to meet, and the scaled density profile at time $t=1 \mathrm{~s}$ and zero temperature is shown in

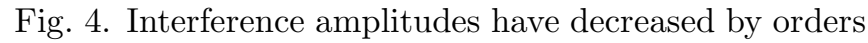
of magnitude when compared to the TF regime, but still tiny signatures are observable. At finite temperature, one then finds virtually no sign of interference anymore, in accordance with expectations based on previous work [28]. As a result, phase coherence is drastically suppressed in the Tonks limit.

Finally, in between the TF and TG limits, one must resort to numerical techniques. After numerical solution of Eq. (6), numerical data for $\rho_{0}(x, t)$ and $\phi_{0}(x, t)$ could be used to solve the eigenproblem corresponding to Eq. (12), and subsequently the interference problem. It is presently unclear whether this program can be carried out in practice. We shall pursue a different route in the next section, where analytical results are given for a related setup employing a $\delta$ potential for the initial axial trap. This allows to access the full crossover between the two limiting cases without approximations. These results qualitatively confirm our above statements obtained in the TF and TG limits.

\section{ALTERNATIVE APPROACH}

So far we have studied interference properties of atomic Bose gases in 1D circular traps after switching off an axial harmonic trap potential. Our treatment had to rely on the local density approximation (LDA) 20] (which requires sufficiently small $\omega_{x}$ ), and, moreover, explicit results were only obtained in the TF and TG limits. To check that the above picture is consistent and robust, in this section we consider a trap potential of the form $V(x, t)=-V_{0} \delta(x) \Theta(-t)$, assuming the circular atom waveguide in Fig. 1 to already contain $N_{0}$ atoms in the absence of $V(x, t)$, so that $\rho_{0}=N_{0} / L$ is the density away from the trap at $t<0$. Let us then imagine that $N \ll N_{0}$ additional atoms are injected $\left(N \propto V_{0}\right.$ is tuned by the trap depth) in the distant past at $x=0$ by adiabatically switching on $V(x, t)$, e.g. from a BEC atom reservoir using a quantum tweezer [41]. At time $t=0$, the trap is switched off, and we again wish to compute the interference signal. This setup may be of relevance to experiments using injection of particles with a quantum tweezer, or for microchip-trapped cold atoms where confinement potentials are quite steep [7]. Importantly, under the condition $N \ll N_{0}$, this interference problem can be solved exactly by virtue of the bosonization method. Using Eq. (5) and the quantum fluctuations defined in Sec. III one now arrives at a standard (uniform and timeindependent) Luttinger liquid Hamiltonian 13] plus the contribution of the trap,

$H(t)=\frac{\hbar u}{2 \pi} \int d x\left[K\left(\partial_{x} \Phi\right)^{2}+K^{-1}(\pi \Pi)^{2}\right]-V_{0} \Theta(-t) \Pi(0, t)$, where $u$ is the sound velocity and $1 \leq K<\infty$ is the dimensionless Luttinger parameter. Both parameters are determined by $g$ and $\rho_{0}$ [13]. In particular, in the TG limit, $K=1$, while $K \gg 1$ in the TF limit. The effect of the trap potential can then be included exactly by the time-dependent unitary transformation $\Pi(x, t) \rightarrow \Pi(x, t)+N \delta(x) \Theta(-t)$, where $N=K V_{0} / \pi \hbar u$ ( $N$ is assumed to be integer) gives the number of added atoms. This allows us to obtain the expansion dynamics $\rho_{0}(x, t)=\rho_{0}+\langle\Pi(x, t)\rangle$ and the interference signal in closed form. From the equation of motion,

$$
\left(-\partial_{t}^{2}+u^{2} \partial_{x}^{2}\right) \Pi(x, t)=N \Theta(-t) \partial_{x}^{2} \delta(x),
$$

for $t<0$ we infer the density $n(x)=\rho_{0}+N \delta(x)$, while for $t>0$ the expanding density profile is

$$
\rho_{0}(x, t)=\rho_{0}+N[\delta(x-u t)+\delta(x+u t)] / 2 .
$$

Apparently, the initial $\delta$-peak in the density is split into two counterpropagating parts, each moving with velocity $u$. Furthermore, the phase $\phi_{0}(x, t)$ is easily found in the form

$$
\phi_{0}(x, t)=\frac{\pi N}{2 K} \Theta(t-|x| / u) .
$$

The interference signal then follows from Eq. (19), with the quantum fluctuations at $x \approx x^{\prime} \approx \pm L / 2$ entering as

$$
F\left(x, x^{\prime}, t\right)=\frac{1}{2 K} \ln \left|\frac{\hbar u}{\pi a_{0} k_{B} T} \sinh \left(\frac{\pi L k_{B} T}{\hbar u}\right)\right|,
$$

where $a_{0}$ is a nonuniversal UV cutoff length limiting the applicability of the continuum model (38). Note that due to our assumption of a constant density $\rho_{0}$ in the absence of the axial trap, the fluctuation factor is now time-independent. Equation (41) implies power-law suppression of the interference signal $\propto 1 / L^{1 / 2 K}$ for short circumference $L<L_{T}$, where

$$
L_{T}=\hbar u / \pi k_{B} T \text {. }
$$

This power law decays quite rapidly in the Tonks limit $(K=1)$, but interference is only weakly suppressed in the TF limit. Moreover, for large circumference $L>L_{T}$, we find an exponential suppression of the interference signal, $\propto \exp \left(-L / 2 K L_{T}\right)$. The findings of the previous section are therefore confirmed from this simple yet exact calculation. There is a smooth crossover between the TF and TG limits, described by the above expressions.

\section{CONCLUSIONS}

In this paper, interference properties of interacting atomic Bose gases in a 1D circular trap have been analyzed. After switching off a trapping potential keeping the atoms in the narrow initial region, the expanding atom clouds meet at the opposite side and may produce 
an interference signal. This signal has been computed for the case of a harmonic initial trap, with explicit results in the Tonks-Girardeau regime and in the ThomasFermi limit, including the effects of thermal fluctuations. Our central conclusion is that many-body interactions strongly suppress phase coherence and interference. In fact, using this setup interference is unlikely to be observed in the strongly interacting Tonks gas. Even in the Thomas-Fermi regime, one needs rather low temperatures and steep initial traps to ensure a significant interference signal. Further support for these conclusions can be drawn from a simple yet exact calculation based on a slightly different setup where one injects a small number of additional atoms into the system, traps them for some time and then studies the expansion and the subsequent interference signal.

\section{Acknowledgments}

We thank R. Graham for valuable discussions. This work has been supported by the DFG under the SFB/TR-12.
[1] M. A. Kasevich, Science 298, 136 (2002).

[2] K. Burnett, P. S. Julienne, P. D. Lett, E. Tiesinga, and C. J. Williams, Nature 416, 225 (2002).

[3] S. L. Rolston and W. D. Phillips, Nature 416, 219 (2002).

[4] R. Dumke, T. Muther, M. Volk, W. Ertmer, and G. Birkl, Phys. Rev. Lett. 89, 220402 (2002).

[5] F. Schreck, L. Khaykovich, K. L. Corwin, G. Ferrari, T. Bourdel, J. Cubizolles, and C. Salomon, Phys. Rev. Lett. 87, 080403 (2001).

[6] S. Richard, F. Gerbier, J. H. Thywissen, M. Hugbart, P. Bouyer, and A. Aspect, Phys. Rev. Lett. 91, 010405 (2003).

[7] W. Hänsel, P. Hommelhoff, T. W. Hänsch, and J. Reichel, Nature 413, 498 (2001).

[8] H. Ott, J. Fortagh, G. Schlotterbeck, A. Grossmann, and C. Zimmermann, Phys. Rev. Lett. 87, 230401 (2001).

[9] A. E. Leanhardt, Y. Shin, A. P. Chikkatur, D. Kielpinski, W. Ketterle, and D. E. Pritchard, Phys. Rev. Lett. 90, 100404 (2003).

[10] K. Bongs, S. Burger, S. Dettmer, D. Hellweg, J. Arlt, W. Ertmer, and K. Sengstock, Phys. Rev. A 63, 031602 (2001).

[11] A. Gorlitz, J. M. Vogels, A. E. Leanhardt, C. Raman, T. L. Gustavson, J. R. Abo-Shaeer, A. P. Chikkatur, S. Gupta, S. Inouye, T. Rosenband, et al., Phys. Rev. Lett. 87, 130402 (2001).

[12] S. Dettmer, D. Hellweg, P. Ryytty, J. J. Arlt, W. Ertmer, K. Sengstock, D. S. Petrov, G. V. Shlyapnikov, H. Kreutzmann, L. Santos, et al., Phys. Rev. Lett. 87, 160406 (2001).

[13] H. Monien, M. Linn, and N. Elstner, Phys. Rev. A 58, 3395 (1998).

[14] E. H. Lieb, R. Seiringer, and J. Yngvason (2003), condmat/0304071.

[15] D. S. Petrov, G. V. Shlyapnikov, and J. T. M. Walraven, Phys. Rev. Lett. 85, 3745 (2000).

[16] R. Graham, Phys. Rev. A 62, 023609 (2000).

[17] A. Csordás and R. Graham, Phys. Rev. A 64, 013619 (2001).

[18] L. Tonks, Phys. Rev. 50, 955 (1936).

[19] M. Girardeau, J. Math. Phys. (N.Y.) 1, 516 (1960).
[20] V. Dunjko, V. Lorent, and M. Olshanii, Phys. Rev. Lett. 86, 5413 (2001).

[21] C. Mora and Y. Castin, Phys. Rev. A 67, 053615 (2003).

[22] J. O. Andersen, U. AlKhawaja, and H. T. C. Stoof, Phys. Rev. Lett. 88, 070407 (2002).

[23] M. Olshanii, Phys. Rev. Lett. 81, 938 (1998).

[24] E. H. Lieb and W. Liniger, Phys. Rev. 130, 1605 (1963).

[25] P. Öhberg and L. Santos, Phys. Rev. Lett. 89, 240402 (2002).

[26] M. D. Girardeau and E. M. Wright, Phys. Rev. Lett. 84, 5239 (2000).

[27] P. Pedri, L. Santos, P. Öhberg, and S. Stringari (2003), cond-mat/0304660.

[28] D. M. Gangardt and G. V. Shlyapnikov, Phys. Rev. Lett. 90, 010401 (2003).

[29] D. S. Petrov, G. V. Shlyapnikov, and J. T. M. Walraven, Phys. Rev. Lett. 87, 050404 (2001).

[30] K. K. Das, M. D. Girardeau, and E. M. Wright, Phys. Rev. Lett. 89, 170404 (2002).

[31] K. K. Das, G. J. Lapeyre, and E. M. Wright, Phys. Rev. A 65, 063603 (2002).

[32] S. Inouye, T. Pfau, S. Gupta, A. P. Chikkatur, A. Gorlitz, D. E. Pritchard, and W. Ketterle, Nature 402, 641 (1999).

[33] D. Hellweg, L. Cacciapuoti, M. Kottke, T. Schulte, K. Sengstock, W. Ertmer, and J. J. Arlt, Phys. Rev. Lett. 91, 010406 (2003).

[34] S. Inouye, M. R. Andrews, J. Stenger, H.-J. Miesner, D. M. Stamper-Kurn, and W. Ketterle, Nature 392, 151 (1998).

[35] W. C. Wu and A. Griffin, Phys. Rev. A 54, 4204 (1996).

[36] T. L. Ho and M. Ma, J. Low. Temp. Phys. 115, 61 (1999).

[37] Y. Kagan, E. L. Surkov, and G. V. Shlyapnikov, Phys. Rev. A 54, 1753 (1996).

[38] Y. Castin and R. Dum, Phys. Rev. Lett. 77, 5315 (1996).

[39] S. Stringari, Phys. Rev. Lett. 77, 2360 (1996).

[40] D. L. Luxat and A. Griffin, Phys. Rev. A. 67, 043603 (2003).

[41] R. B. Diener, B. Wu, M. G. Raizen, and Q. Niu, Phys. Rev. Lett. 89, 070401 (2002). 